\title{
Gene expression and promoter polymorphisms of DNA methyltransferase 3B in nasopharyngeal carcinomas in Taiwanese people: A case-control study
}

\author{
KAI-PING CHANG ${ }^{1,2}$, SHENG-PO HAO ${ }^{1}$, NGAN-MING TSANG ${ }^{3}$, YU-LIANG CHANG ${ }^{4}$, \\ MING-HUEI CHENG ${ }^{5}$, CHUN-TING LIU ${ }^{6}$, YUN-SHIEN LEE ${ }^{6,7}$, CHIA-LUNG TSAI $^{8}$, \\ TA-JEN LEE ${ }^{1}$, TZU-HAO WANG ${ }^{6,8}$ and CHI-NEU TSAI ${ }^{2}$
}

\begin{abstract}
${ }^{1}$ Department of Otolaryngology - Head and Neck Surgery, Chang Gung Memorial Hospital; ${ }^{2}$ Graduate Institute of Clinical Medical Sciences, Chang Gung University; ${ }^{3}$ Departments of Radiation Oncology, ${ }^{4}$ Oral and Maxillofacial Surgery, and ${ }^{5}$ Plastic and Reconstructive Surgery, Chang Gung Memorial Hospital; ${ }^{6}$ Genomic Medicine Research Core Laboratory, Chang Gung Memorial Hospital, Kwei-Shan, Tao-Yuan; ${ }^{7}$ Department of Biotechnology, Ming-Chuan University, Tao-Yuan;

${ }^{8}$ Department of Obstetrics and Gynecology, Chang Gung Memorial Hospital, Kwei-Shan, Tao-Yuan, Taiwan
\end{abstract}

Received May 14,2007; Accepted August 16, 2007

\begin{abstract}
Overexpression of the DNA methyltransferase 3B (DNMT3B) gene and its effect on carcinogenesis has been demonstrated for various types of cancer. Recently, three single nucleotide polymorphisms (SNPs) of the DNMT3B promoter region, C46359T $(-149 \mathrm{C}>\mathrm{T}),-283 \mathrm{~T}>\mathrm{C}$, and $-579 \mathrm{G}>\mathrm{T}$ have also been reported to be stratification markers that can predict an individual's susceptibility to cancers. In this study, we analyzed expression of $D N M T 3 B$ in nasopharyngeal carcinoma (NPC) specimens and did not find elevated levels of DNMT3B in tumors using cDNA microarray analysis and RT-PCR. Meanwhile, 259 NPC patients and 250 controls were genotyped for the above three SNPs using a MALDI-TOF based minisequencing method. For C46359T $(-149 \mathrm{C}>\mathrm{T})$, only the T/T genotype was found to be present in both patient and control groups (100\% frequency). The frequency of the genotypes, -283CC, -283CT and -283TT, amongst NPC patients versus controls was, respectively, $86.1 \%$ versus $84.0 \%, 13.5 \%$ versus $15.6 \%$, and $0.4 \%$ versus $0.4 \%(\mathrm{P}=0.589)$. The allele frequency, -597TT, -597GT and -597GG, for patients versus controls was, respectively, $87.3 \%$ versus $84.8 \%, 12.0 \%$ versus $15.2 \%$, and $0.8 \%$ versus $0(\mathrm{P}=0.501)$. The distribution of SNPs among cancer patients either featuring or not featuring cervical metastasis also did not reveal any significant difference. In conclusion, our data indicate that neither overexpression of $D N M T 3 B$ nor the presence of three DNMT3B SNPs are associated with NPC, which suggests that DNMT3B might
\end{abstract}

Correspondence to: Dr Chi-Neu Tsai, Graduate Institute of Clinical Medical Sciences, Chang Gung University, 259 Wen-Hwa $1^{\text {st }} \mathrm{Rd}$, Kwei-Shan, Tao-Yuan, Taiwan

E-mail: pink7@mail.cgu.edu.tw

Key words: nasopharyngeal carcinoma, DNA methyltransferase 3B, single nucleotide polymorphism not play a role in hypermethylation of many tumor suppressor genes during carcinogenesis of NPC.

\section{Introduction}

CpG methylation patterns are established and maintained during development by DNA methyltransferase 1 (DNMT1) and DNMT3 families in mammalian species (1). Global epigenetic alterations such as 'globally hypomethylated; gene specific hypermethylated in DNA' have been found in cancer cells, which has highlighted the idea that DNA methylation plays a significant role in development and progression of cancers (2). Indeed, reports have shown that cell cycle control, apoptosis-related, tumor suppressor and DNA repair genes are hypermethylated in several kinds of cancer cells (2). DNA methylation is mediated by DNA methyltransferases including DNMT1, DNMT3A and DNMT3B which cooperatively establish and maintain genomic methylation patterns (3). DNMT1 is considered to be a maintenance DNA methyltransferase due to the fact that it preferentially methylates hemimethylated DNA after DNA replication; whereas DNMT3A and DNMT3B function as de novo methyltransferases, which methylate unmethylated and hemi-methylated DNA with equal efficiency (3).

Among these DNMTs, overexpression of DNMT3B was found to be present in several tumors, demonstrating that DNMT3B plays an important role in tumorigenesis (4-7). Recently, three single nucleotide polymorphisms (SNPs) of the DNMT3B promoter [C46359T $(-149 \mathrm{C}>\mathrm{T}),-283 \mathrm{~T}>\mathrm{C}$ and $-579 \mathrm{G}>\mathrm{T}$ ] were reported to be crucial for the promoter's activity. This results in an involved individual's predisposition to certain cancers; including lung cancer, prostate cancer, hereditary nonpolyposis colorectal cancer (HNPCC) and acute leukemia (8-11). Nevertheless, the biological significance of these three DNMT3B SNPs is somewhat controversial as regards individuals of differing ethnicity, and persons with different types of tumors (12-14). 
Nasopharyngeal carcinoma (NPC) is a common cancer in the southeast region of Asia including Taiwan (15). Although Epstein-Barr virus has been reported to be associated with NPC, certain other factors, such as environmental or genetic factors, still appear crucial for the development and/or progression of NPC (16). In some epigenetic studies, hypermethylation of the promoters of many critical tumor suppressor genes has been shown in NPC (17-19). Latent membrane protein-1, an oncoprotein derived from the Epstein-Barr virus, can activate the promoter of DNMT1 via the JNK-AP-1 pathway and then induce the downregulation of the E-cadherin gene which increases the migration capability of NPC cells $(20,21)$. However, the relative significance of the expression pattern of DNMT3B and these novel SNPs with regards to the genetic susceptibility of an individual to NPC in Taiwan, to the best of our knowledge, has not been determined. Therefore, the aim of this hospital-based case-control study was to examine the expression patterns of DNMT3B in NPC specimens and pericancerous normal counterparts, and determine whether these three novel SNPs of DNMT3B are actually risk factors for NPC within the Taiwanese population. The genotype of each of these three DNMT3B SNPs was determined using a matrix-assisted laser desorption/ionization time-of-flight/ mass spectrometry (MALDI-TOF/MS)-based mini-sequencing method (22).

\section{Materials and methods}

Study population. This study was approved by the Institutional Review Board of the Chang Gung Memorial Hospital. Subjects were recruited and enrolled into the study from consecutive patients included in an ongoing molecular epidemiological study being conducted within the Department of Otolaryngology - Head and Neck Surgery at Chang Gung Memorial Hospital, Tao-Yuan, Taiwan. All cases were newly diagnosed, previously untreated patients with NPC. All had their condition histopathologically confirmed between September 1990 and November 2005, inclusively. No restrictions applied regarding participant age, gender, histological classification, and/or tumor stage. Control subjects were derived from a pool of volunteers attending the hospital as part of a routine health-examination program, including individuals presenting with otolaryngological-related, non-neoplastic diseases. All NPC patients enrolled in this study underwent, prior to their treatment, routine diagnostic work-ups including head-and-neck computed tomography and/or magnetic resonance imaging, chest x-ray, abdominal ultrasonography, and a bone scan. Those NPC patients, who had a positive nodal status as revealed by radiological examination conducted at the time of presentation, were defined as NPC cases featuring cervical metastasis. All patients recruited for this study provided written informed consent for study participation and blood-sample collection.

cDNA microarray analysis. Total RNA was extracted from frozen paired tumor and pericancerous normal tissues using the RNAzol B reagent (Tel-Test Inc., Friendwood, TX), and further purified and concentrated with the RNeasy cleanup kit (Qiagen Inc., Valencia, CA). RNA quality and quantity was evaluated with a Bioanalyzer 2100 (Agilent Technologies, Palo Alto, CA). To minimize the effects of normal stromal tissue contamination on gene expression profiling of the tumor tissues, 19 paired samples having a minimum of $80 \%$ tumor cells in all tumor samples (as determined by our pathologist from the corresponding hematoxylin/eosin-stained paraffin sections) were selected for cDNA microarray analysis.

cDNA microarray analysis was performed using total RNA extracted from NPC and paired normal tissues. Information regarding the GMRCL Human 15K set, Version 2 microarray chips used in this study can be downloaded from <http:// www.cgmh.org.tw/intr/intr2/c32a0/chinese/corelab_intro/ genetics/index_1.htm $>$ in the Minimum Information About a Microarray Experiment (MIAME) format. Briefly, $2 \mu \mathrm{g}$ total RNA was used for labeling and hybridization with the 3DNA 300Array detection kit (Genisphere, Hatfield, PA) according to the manufacturer's protocol. The experiment was replicated under the dye-swapping microarray strategy in an effort to minimize statistical variances in the data. After hybridization and washing, slides were scanned with a ChipReader confocal scanner (Virtek, Canada) and spot and background intensities were acquired using GenePix Pro 4.1 software (Molecular Devices, Sunnyvale, CA). For within-slide normalization, the local weighted regress (LOWESS) method was used. We averaged four replicated log ratios of each gene (two from duplicates on the slide and two others from the dye-swapping replicate) and performed Student's t-tests on each gene between the indicated groups. Stringent criteria were used to define the statistical significance of each observed change in gene expression, using $\mathrm{F}$ statistics $(\mathrm{p}<0.001)$, with significance confirmed over 1,000,000 random permutations.

Reverse transcription-polymerase chain reaction (RT-PCR) of $D N M T 3 B$. One $\mu \mathrm{g}$ of total RNA purified from tissues samples was reverse transcribed using reverse transcriptor first-strand cDNA synthesis kits (Roche) in a total volume of $25 \mu 1$. RT-PCR amplification reactions of DNMT3B and PCNA were performed with $100 \mathrm{ng}$ cDNA, 10\% DMSO, $100 \mu \mathrm{M}$ deoxynucleotide triphosphates, Taq DNA polymerase (Roche), and $1 \mu \mathrm{M}$ primers. The RT-PCR primers for DNMT3B isoforms were as follows: DNMT3B catalytic sense, 5'-AAG CCC ATG CAA TGA TCT CTC TAA CG-3' and antisense, 5'-CAC GTC CGT GTA GTG AGC AGG GAA GC-3'; and human PCNA sense, 5'-CAA CTT GGA AT CCA GAA CAG GAG TAC AGC-3' and antisense, 5'-GGG TAC ATC TGC AGA CATR ACT GAG TGT CA-3'. The primer and PCR condition was as previously described (23).

DNMT3B genotyping by matrix-assisted laser desorption/ ionization time-of-flight (MALDI-TOF)-based mini-sequencing analysis. Genomic DNA was extracted from peripheral blood lymphocytes according to the manufacturer's recommendations (Nucleobond, Macherey-Nagel). The DNMT3B -283T/C, 579G/T, C46359T polymorphisms were determined by a MALDI-TOF based mini-sequencing genotyping method as previously described $(24,25)$. The primer sets used for amplification of each SNP region were as described previously (26). In brief, the PCR reactions were performed in a total of $25 \mu \mathrm{l}$ containing $200 \mathrm{ng}$ genomic DNA, $25 \mathrm{pM}$ each primer, $0.2 \mathrm{mM}$ dNTPs, $75 \mathrm{mM}$ Tris- $\mathrm{HCl}$ (pH 9.0), $15 \mathrm{mM}$ ammonium sulfate, $0.1 \mu \mathrm{g} / \mathrm{BSA}, 2.5 \mathrm{mM} \mathrm{MgCl}{ }_{2}, 1 \mathrm{M}$ betaine and 1 unit of Fast-start Taq polymerase (Roche), which was initiated for 
$95^{\circ} \mathrm{C}$ for $5 \mathrm{~min}$, followed by 40 cycles of $95^{\circ} \mathrm{C}$ for $45 \mathrm{sec}$, $50^{\circ} \mathrm{C}$ for $45 \mathrm{sec}$ and $72^{\circ} \mathrm{C}$ for $45 \mathrm{sec}$, and finally extended for $10 \mathrm{~min}$ at $72^{\circ} \mathrm{C}$. The unincorporated dNTPs and primers were removed automatically by MAPIIA (genepure PCR purification system; Brucker, Bremen, Germany). The purified PCR products were used to conduct mini-sequencing reactions by individual mini-sequencing primers as we described previously (26). These were carried out in $20 \mu 1$ of solution containing $50 \mathrm{ng}$ PCR product and $1 \mu \mathrm{l}$ of 10 pmol minisequencing primer, $0.5 \mu 1$ of $1 \mathrm{mM}$ of ddNTPs/dNTP mixture (for $-283 \mathrm{~T} / \mathrm{C}$, dA ddC ddG; -579G/T, dT ddG; C46359, dC ddA ddT), $0.5 \mathrm{U}$ of Thermo Sequenase DNA polymerase (Amersham Biosciences), and $2 \mu 1$ reaction buffer provided by the manufacturer. The reaction was carried out in a multiblock system (MBS) thermocycler (ThermoHybaid) with an initial denaturation step at $96^{\circ} \mathrm{C}$ for 1 min followed by 50 cycles of $96^{\circ} \mathrm{C}$ for $15 \mathrm{sec}, 50^{\circ} \mathrm{C}$ for $15 \mathrm{sec}, 60^{\circ} \mathrm{C}$ for $100 \mathrm{sec}$, and then $96^{\circ} \mathrm{C}$ for $30 \mathrm{sec}$. The mini-sequencing reaction products were purified by single-strand binding beads automatically via MAPIIA (Brucker) and analyzed by MALTI-TOF MS. Prior to MALDI-TOF MS analysis, samples were mixed with $0.5 \mu \mathrm{l}$ of matrix solution $(50 \mathrm{mg} / \mathrm{ml} \mathrm{3-hydropicolinic}$ acid in a 4:5:1 mixture of water, acetonitrile and $50 \mathrm{mg} / \mathrm{ml}$ diammonium citrate) and spotted on a 384-well teflon sample plate (PerSeptive Biosystems). MALDI-TOF mass spectra were acquired using a Brucker Autoflex MALDI-TOF (Brucker) and AutoXecute software as validating tools for genotyping data. To confirm the results of MALDI-TOF, 1/10 of the PCR products were randomly selected for DNA auto-sequencing analysis (ABI autosequencer 3730 ) by $3 \mathrm{~b}-1$ sequencing primer (5'-AATTT GAAAT CGCTC GGAGC CTC-3'), 3b-2 sequencing primer (5'-GACCT GGAGC TGTTT GTGGT TC-3') or 3b-3 sequencing primer (5'-ACCAC CTCTA TTCCA AGAAC TG-3'), respectively (26).

Statistical analysis. Cases and controls were compared using Student's t-test for continuous variables and the $\chi^{2}$ test for categorical variables. The Hardy-Weinberg equilibrium was tested with a goodness-of-fit $\chi^{2}$ test with one degree of freedom, to compare the observed genotype frequencies with the expected genotype frequencies among the subjects. Unconditional logistic regression analysis was used to calculate odds ratios (ORs) and $95 \%$ confidence intervals (CIs), with adjustment for possible confounders (sex as a nominal variable and age as continuous variables). All analyses were performed using Statistical Analysis Software for Windows, version 8.12 (SAS institute, Gary, NC, USA).

\section{Results}

Subjects were enrolled between September 1990 and November 2005 in Chang Gung Memorial Hospital without restriction in age, sex, histological, or stage restrictions. Case-control studies included 259 patients newly diagnosed with NPC and 250 controls. The characteristics of all enrolled individuals are shown in Table I. There was no statistically significant difference in age and gender between NPC cases and controls. The genotypes of the three DNMT3B SNPs were determined by means of a MALDI-TOF-based mini-sequencing method $(24,25)$, the primer sets used for generating three separate
Table I. The clinicopathological characteristics of study subjects.

\begin{tabular}{lccc}
\hline Characteristics & NPC & Control & P-value \\
\hline Gender & & & \\
Female & 74 & 79 & 0.517 \\
Male & 185 & 171 & \\
Age (years) & & & \\
Range & $13.0-87.9$ & $16.3-78.2$ & 0.459 \\
Mean \pm SD & $47.7 \pm 12.0$ & $48.6 \pm 13.5$ & \\
Pathology, n (\%) & & & \\
SCC & $9(3.5 \%)$ & & \\
NKC & $104(40.2 \%)$ & & \\
UC & $130(50.2 \%)$ & & \\
Not specified & $16(6.2 \%)$ & & \\
Clinical stage, n $(\%)$ & & & \\
I & $34(13.1 \%)$ & & \\
II & $86(33.2 \%)$ & \\
III & $75(28.9 \%)$ & \\
IV & $64(24.7 \%)$ & \\
Total & 259 & 250 \\
\hline
\end{tabular}

SCC, squamous cell carcinoma; NKC, non-keratinizing carcinoma; $\mathrm{UC}$, undifferentiated carcinoma.

DNA fragments covering each of the three DNMT3B SNPs, the mini-sequencing primer, and the molecular weight of mini-sequencing products are as we described previously (26). The PCR products were randomly selected to determine their DNA sequences by auto-sequencing analysis. The results of DNA sequences of the three DNMT3B SNPs confirmed the genotyping results (data not shown).

Contrary to what has been reported in several previous studies, we observed that only the T/T genotype (C46359T, $-149 \mathrm{C}>\mathrm{T}$ ) was found to be present in the DNA of individuals from both study groups (100\% frequency), whereas the $\mathrm{C} / \mathrm{C}$ and $\mathrm{C} / \mathrm{T}$ genotype $(\mathrm{C} 46359 \mathrm{~T},-149 \mathrm{C}>\mathrm{T}$ ) was not found to be present in either of the two study groups. Our data suggest that there is no association between SNP (C46359T) of the $D N M T 3 B$ promoter and NPC risk in a Taiwanese population, similar to a finding for gastric cancer in a Japanese population (12).

The frequency of appearance of the genotypes, -283CC, $-283 \mathrm{CT}$ and -283TT, amongst NPC patients versus controls was, respectively, $86.1 \%$ versus $84.0 \%, 13.5 \%$ versus $15.6 \%$, and $0.4 \%$ versus $0.4 \%$. The frequency of appearance of the two genotypes, -597TT, -597GT and $-597 \mathrm{GG}$, for patients versus controls was, respectively, $87.3 \%$ versus $84.8 \%, 12.0 \%$ versus $15.2 \%$, and $0.8 \%$ versus 0 (Table II). Our data show that the incidence of these two DNMT3B SNPs for individuals afflicted with NPC did not differ significantly from corresponding values for controls $(-283 \mathrm{~T}>\mathrm{C}, \mathrm{P}=0.589 ;-597 \mathrm{G}>\mathrm{T}$, $\mathrm{P}=0.501$ ) (Table II). 
Table II. DNMT3B genotype and allele frequency among NPC cases and controls.

\begin{tabular}{|c|c|c|c|c|c|c|c|c|}
\hline & \multicolumn{3}{|c|}{$-283 \mathrm{~T}>\mathrm{C}$ genotype } & \multirow[b]{2}{*}{ P-value ${ }^{a}$} & \multicolumn{3}{|c|}{$-579 \mathrm{G}>\mathrm{T}$ genotype } & \multirow[b]{2}{*}{ P-value } \\
\hline & $\mathrm{TT}$ & $\mathrm{TC}$ & $\mathrm{CC}$ & & $\mathrm{GG}$ & GT & $\mathrm{TT}$ & \\
\hline Control & $1(0.4 \%)$ & $39(15.6 \%)$ & $210(84.0 \%)$ & 0.589 & 0 & $38(15.2 \%)$ & $212(84.8 \%)$ & 0.501 \\
\hline Case & $1(0.4 \%)$ & $35(13.5 \%)$ & $223(86.1 \%)$ & & $2(0.8 \%)$ & $31(12.0 \%)$ & $226(87.3 \%)$ & \\
\hline
\end{tabular}

${ }^{\mathrm{a} C a l c u l a t e d}$ by chi-square between TT+TC and CC. ${ }^{\mathrm{b}} \mathrm{Calculated}$ by chi-square between GG+GT and TT.

Table III. Crude and adjusted ORs for all NPC cases and cases with cervical metastasis. ${ }^{\mathrm{a}}$

\begin{tabular}{lcccr}
\hline Genotype & All cases & P-value & CM & P-value \\
\hline -283T $>$ C genotype & & & & \\
CC (reference) & 1.0 & 0.506 & $0.486(0.218-1.083)$ & 0.078 \\
TC+TT crude & $0.848(0.520-1.381)$ & 0.493 & $0.500(0.219-1.143)$ & 0.100 \\
Adjusted & $0.843(0.517-1.374)$ & & & \\
-579G $>$ T genotype & & & $0.635(0.274-1.471)$ & 0.289 \\
TT (reference) & 1.0 & 0.424 & $0.649(0.275-1.532)$ & 0.324 \\
GT+GG crude & $0.815(0.493-1.347)$ & 0.431 & \\
Adjusted & $0.817(0.494-1.351)$ & &
\end{tabular}

${ }^{a}$ ORs (95\% CIs) and corresponding P-values were calculated by logistic regression, adjusted for age and sex. CM, patients with cervical metastasis, adjusted for age, sex, and pathological classification.

The risk of suffering NPC and contracting cervical metastasis appears to relate to the presence or absence of either of the two DNMT3B genotypes $-283 \mathrm{~T}>\mathrm{C}$ and $-579 \mathrm{G}>\mathrm{T}$, the odds ratios (ORs) for which were also analyzed (Table III). ORs and their $95 \%$ CIs were calculated using the more-common homozygous genotype as the reference group (-283CC and -579TT genotypes, respectively). When comparing the results for the patient group with the corresponding results for the control group, the ORs for NPC for the -283 TT and the TC genotype combined, and the ORs for NPC for the -579 GT genotype combined with the GG genotype were lower for the patient group than was the case for the control group, although such differences did not prove to be statistically significant (respectively, adjusted $\mathrm{OR}=0.843$, 95\% $\mathrm{CI}=0.517-1.374$, $\mathrm{P}=0.493$; adjusted $\mathrm{OR}=0.817,95 \% \mathrm{CI}=0.494-1.351, \mathrm{P}=0.431$ ) (Table III). Of the 259 NPC patients, 160 (61.8\%) had cervical metastasis upon radiological examination conducted prior to treatment for NPC, although none of these 160 cervicalmetastasis patients were found to show any evidence of distant metastasis during systemic work-up prior to their treatment. Again, the ORs for cervical metastasis for the two genotypes -283 TT and TC combined, and for the -579 GT and GG genotypes combined were observed to be lower within the patient group, but again were not statistically significant when compared with the corresponding results for the reference group (adjusted $\mathrm{OR}=0.500,95 \% \mathrm{CI}=0.219-1.143, \mathrm{P}=0.100$; adjusted $\mathrm{OR}=0.649,95 \% \mathrm{CI}=0.275-1.532, \mathrm{P}=0.324$, respectively) (Table III). Our data thus reveal that there are no significant differences between NPC patients and controls as regards to the frequency or presence of any or all of these three DNMT3B SNPs or the likelihood of the occurrence of carcinogenesis and/or cervical metastasis for NPC patients, at least amongst a Taiwanese population.

Since a previous paper showed that polymorphisms in $D N M T 3 B$ promoters increase promoter activity of $D N M T 3 B$, the transcripts of $D N M T 3 B$ were also examined by cDNA microarray analysis in 19 NPC biopsies and their normal counterparts whose $D N M T 3 B$ genotype had been determined. Most of them were -283CC/-597GG; only one was -283CT/ -597GT. As regards DNMT3B expression profiles on cDNA microarrays, we found that the fold change of DNMT3B mRNA signal ratio between each paired NPC tumor and pericancerous normal tissue ranged from 0.420 to 1.562 and there was no significant differential expression of $D N M T 3 B$ mRNA (0.957 $\pm 0.276 ; \mathrm{P}=0.314$ ) (Fig. 1A). Elevated levels of $D N M T 3 B$ were not found in NPC biopsies as compared with their pericancerous normal tissue. Among 19 NPC biopsies, some did not show expression of DNMT3B $(\mathrm{n}=5)$ and even showed a tendency toward down-regulation of $D N M T 3 B$ $(\mathrm{n}=8)$ (Fig. 1A). We confirmed the expression of DNMT3B by real-time quantitative reverse transcription-polymerase chain reaction (RT-PCR); the data were consistent with results of cDNA microarray profiling (data not shown). Previous reports indicated that several isoforms of $D N M T 3 B$ were over- 
(A)

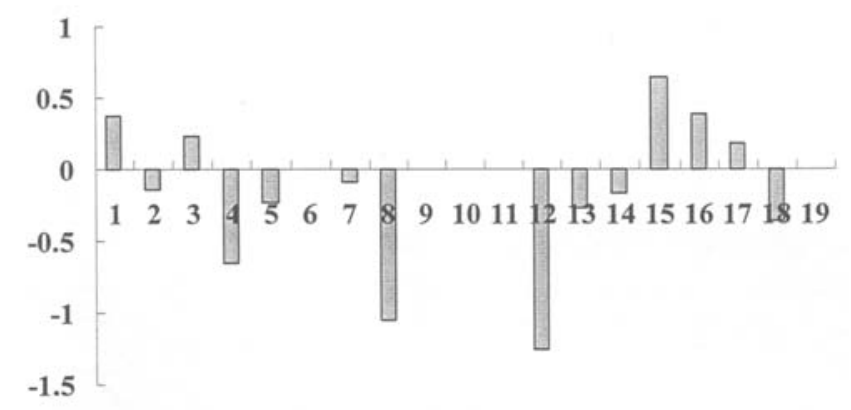

(B)

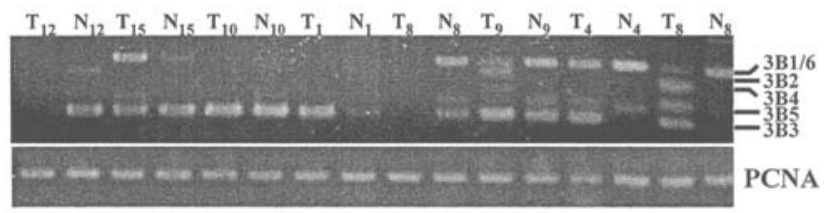

Figure 1. Relative gene expression level of DNMT3B in NPC biopsies. (A) The expression level of DNMT3B was examined in 19 NPC biopsies and their normal counterparts by cDNA microarray. For each of the NPC biopsies, the expression of DNMT3B in the NPC biopsy or normal counterpart was first normalized by expression level of GAPDH, and the fold change of DNMT3B mRNA signal ratio (log) was shown for each pair: NPC tumor divided by pericancerous normal tissue after normalization via GAPDH. The $\mathrm{y}$-axis shows the relative gene expression level; the $\mathrm{x}$-axis shows these 19 NPC biopsies. (B) Expression of DNMT3B transcripts in NPC tissues and normal counterparts was examined by RT-PCR. The PCR product was separated on $2 \%$ agarose gel and the molecular weights of each DNMT3B isoform are indicated by arrowheads. M, marker; T, tumor; N, normal counterpart of each tumor. Expression of PCNA was used as an internal control in the RT-PCR experiment.

expressed in tumor cells including $D N M T 3 B 4$ and $\triangle-D N M T 3 B$. Thus, the isoforms of $D N M T 3 B$ were examined by consensus primer sets as previously described (23). Some of the NPC biopsies did not express any DNMT3B isoforms as compared with their normal counterparts (Fig. 1B; T12 and T8). The predominant form of $D N M T 3 B$ in NPC tumors and normal tissues was DNMT3B; DNMT3B4 was observed in only one tumor (Fig. 1A; T6). Most of them showed expression of different $D N M T 3 B$ isoforms between normal and tumor (Fig. 1B; T2/N2, T6/N6, T7/N7, and T8/N8). Neither overexpression of DNMT3B nor DNMT3B4 was found in NPC tumors as compared with their normal counterparts.

\section{Discussion}

Overexpression of $D N M T 3 B$ has been found in several cancers, including lung cancer, endometrial cancer, breast cancer, and oral cancer, by using either immunohistochemical analysis or real-time quantitative reverse transcriptase-polymerase chain reaction (QRT-PCR) (4-7). Using immunohistochemical analysis, DNMT1, DNMT3A and DNMT3B proteins were found to be highly expressed in a coordinate manner in lung cancers, particularly in smokers (5). Also, overexpression of $D N M T 3 B$ was found to be an independent prognostic marker for breast cancer, especially for estrogen receptor $\alpha$-positive breast cancers (27). In addition, DNMT3B overexpression was associated with poor relapse-free survival in the subgroup of patients who received adjuvant hormone therapy (7). However, a role for $D N M T 3 B$ in NPC is not established. To investigate a role for $D N M T 3 B$ in NPC, we analyzed the expression of $D N M T 3 B$ between NPC tumors and their pericancerous counterparts by cDNA microarray and QRT-PCR. Unlike the cancers mentioned above, the expression of DNMT3B did not show significant differences between NPC tumors and their normal counterparts (Fig. 1A). In previous studies, the predominant isoforms of $D N M T 3 B$ had been identified as contributing to human carcinogenesis $(28,29)$. Overexpression of $D N M T 3 B 4$, a splice variant of $D N M T 3 B$, which may lack DNA methyltransferase activity and compete with DNMT3B3 for targeting to pericentromeric satellite regions, results in DNA hypomethylation in these regions, even in precancerous stages, and plays a critical role in human hepatocarcinogenesis by inducing chromosomal instability (28). Also, a novel $D N M T 3 B$ subfamily, termed $\triangle$-DNMT3B, has been shown to be the major expression form of $D N M T 3 B$ in non-small cell lung cancer and may play an important role in the development of aberrant promoter methylation during lung tumorigenesis. To further identify the predominant isoforms of DNMT3B in NPC we analyzed QRT-PCR results. We observed DNMT3B4 in only one tumor sample (Fig. 1B; T6). Furthermore, expression of DNMT3B was not detected in some NPC tumors as compared with their normal counterparts (Fig. 1B; T12 and T8). In other words, unlike the effects of $D N M T 3 B$ on many types of malignancies, neither $D N M T 3 B$ overexpression nor DNMT3B4 appear to have a role in NPC tumors.

Recently, a single nucleotide polymorphism of the DNMT3B promoter $(\mathrm{C} 46359 \mathrm{~T},-149 \mathrm{C}>\mathrm{T})$ was reported to be crucial for its promoter activity, although the results were conflicting. However, these results showed that persons with this SNP have a predisposition to certain cancers including lung, prostate, and acute leukemia $(9,10,11)$. However, other studies showed conflicting results, that the C46359T polymorphism of DNMT3B $(-149 \mathrm{C}>\mathrm{T})$ cannot be used as a stratification marker for prediction of susceptibility to breast and lung tumors, Caucasian HNPCC and gastric cancer $(8,12,14)$. In our study, the CT-genotype was not found in either control or NPC groups from Taiwan. Our finding is very similar to findings from several recent reports, which showed that the polymorphism in C46359T of DNMT3B is not significant either in gastric cardiac adenocarcinoma, acute leukemia patients of Chinese populations and gastric cancer patients in Japanese populations $(9,12,14)$. Moreover, the T/T alleles of C46359T were found in $100 \%$ of cases in both NPC patients and healthy controls, which is consistent with other studies in Asian populations which show that more than $95 \%$ of alleles in $\mathrm{C} 46359 \mathrm{~T}$ were $\mathrm{T} / \mathrm{T}(9,12,14)$. These findings are distinct from the results reported for Caucasian populations and might indicate that C46359T of DNMT3B is unable to be a useful stratification marker to predict susceptibility or survival differences of NPC in populations in Taiwan. 
Besides C46359T, -283T>C (-283 bp from the transcription start site of exon $1 \mathrm{~A})$ and $-579 \mathrm{G}>\mathrm{T}(-579 \mathrm{bp}$ from the transcription start site of exon $1 \mathrm{~B})$ of $D N M T 3 B$ were investigated recently because expression of the $D N M T 3 B$ gene is regulated via different promoters, which exist in different exons (exons $1 \mathrm{~A}$ and $1 \mathrm{~B})$ with different $\mathrm{CpG}$ content $(30,31)$. A previous study suggested that the $-283 \mathrm{C}$ allele (within exon $1 \mathrm{~A}$ of $D N M T 3 B$ ) may create a potential $\mathrm{Sp} 1$ responsive element to enhance promoter activity nearly two fold over -283T, and demonstrated that -283TT was associated with a significantly decreased risk of adenocarcinoma of the lung with an adjusted odds ratio of 0.48 as compared with -283CC (8). Therefore, the diversity in DNMT3B SNPs in different ethnic populations or in different tumor types remains to be elucidated.

In conclusion, our study shows that DNMT3B has only trivially small effects on NPC carcinogenesis because it has been shown not to be overexpressed in NPC specimens. Also, the three reported DNMT3B SNPs (C46359T, haplotype $-283 \mathrm{~T}>\mathrm{C}$ and $-579 \mathrm{G}>\mathrm{T}$ ) were not associated with higher risk of susceptibility to NPC. Furthermore, an impact of these DNMT3B SNPs on tumor metastasizing tendencies was not observed in populations of Taiwan. Consequently, DNMT3B should not be considered to have a major role in the phenomenon of hypermethylation of many vital genes during NPC carcinogenesis.

\section{Acknowledgements}

This study was supported by grants from the Chang Gung Memorial Hospital (no. CMRPG33117 and CMRPG32090) and the National Science Council of Taiwan (no. NSC-93231-B186-006 and NSC 95-2314-B-182A-105).

\section{References}

1. Bestor TH: The DNA methyltransferases of mammals. Hum Mol Genet 9: 2395-2402, 2000.

2. Momparler RL and Bovenzi V: DNA methylation and cancer. J Cell Physiol 183: 145-154, 2000.

3. Robertson KD: DNA methylation and human disease. Nat Rev Genet 6: 597-610, 2005.

4. Jin F, Dowdy SC, Xiong Y, Eberhardt NL, Podratz KC and Jiang SW: Up-regulation of DNA methyltransferase 3B expression in endometrial cancers. Gynecol Oncol 96: 531-538, 2005.

5. Lin RK, Hsu HS, Chang JW, Chen CY, Chen JT and Wang YC: Alteration of DNA methyltransferases contributes to $5^{\prime} \mathrm{CpG}$ methylation and poor prognosis in lung cancer. Lung Cancer 55: 205-213, 2007.

6. Yakushiji T, Uzawa K, Shibahara T, Noma H and Tanzawa H: Over-expression of DNA methyltransferases and CDKN2A gene methylation status in squamous cell carcinoma of the oral cavity. Int J Oncol 22: 1201-1207, 2003.

7. Girault I, Tozlu S, Lidereau R and Bieche I: Expression analysis of DNA methyltransferases 1,3A, and 3B in sporadic breast carcinomas. Clin Cancer Res 9: 4415-4422, 2003.

8. Lee SJ, Jeon HS, Jang JS, et al: DNMT3B polymorphisms and risk of primary lung cancer. Carcinogenesis 26: 403-409, 2005.

9. Li Y, Dai Y, Wu SL, Pei P, Cao XH and Pu DF: The C46359T polymorphism of DNMT3B promoter gene and pathogenesis of acute leukemia. Zhonghua Nei Ke Za Zhi 44: 588-591, 2005.

10. Shen H, Wang L, Spitz MR, Hong WK, Mao L and Wei Q: A novel polymorphism in human cytosine DNA-methyltransferase$3 \mathrm{~B}$ promoter is associated with an increased risk of lung cancer. Cancer Res 62: 4992-4995, 2002.
11. Singal R, Das PM, Manoharan M, Reis IM and Schlesselman JJ: Polymorphisms in the DNA methyltransferase $3 \mathrm{~b}$ gene and prostate cancer risk. Oncol Rep 14: 569-573, 2005.

12. Aung PP, Matsumura S, Kuraoka K, et al: No evidence of correlation between the single nucleotide polymorphism of $D N M T 3 B$ promoter and gastric cancer risk in a Japanese population. Oncol Rep 14: 1151-1154, 2005.

13. Montgomery KG, Liu MC, Eccles DM and Campbell IG: The DNMT3B C->T promoter polymorphism and risk of breast cancer in a British population: a case-control study. Breast Cancer Res 6: 390-394, 2004.

14. Wang YM, Wang R, Wen DG, et al: Single nucleotide polymorphism in DNA methyltransferase 3B promoter and its association with gastric cardiac adenocarcinoma in North China. World J Gastroenterol 11: 3623-3627, 2005

15. Hsu MM and Tu SM: Nasopharyngeal carcinoma in Taiwan. Clinical manifestations and results of therapy. Cancer 52: 362-368, 1983.

16. Wei WI and Sham JS: Nasopharyngeal carcinoma. Lancet 365 : 2041-2054, 2005.

17. Tan SH, Ida H, Goh BC, Hsieh W, Loh M and Ito Y: Analyses of promoter hypermethylation for RUNX3 and other tumor suppressor genes in nasopharyngeal carcinoma. Anticancer Res 26: 4287-4292, 2006.

18. Kwong J, Lo KW, To KF, Teo PM, Johnson PJ and Huang DP: Promoter hypermethylation of multiple genes in nasopharyngeal carcinoma. Clin Cancer Res 8: 131-137, 2002.

19. Lo KW, Kwong J, Hui AB, et al: High frequency of promoter hypermethylation of RASSF1A in nasopharyngeal carcinoma. Cancer Res 61: 3877-3881, 2001.

20. Tsai CL, Li HP, Lu YJ, et al: Activation of DNA methyltransferase 1 by EBV LMP1 involves c-Jun NH(2)-terminal kinase signaling. Cancer Res 66: 11668-11676, 2006.

21. Tsai CN, Tsai CL, Tse KP, Chang HY and Chang YS: The Epstein-Barr virus oncogene product, latent membrane protein 1, induces the downregulation of E-cadherin gene expression via activation of DNA methyltransferases. Proc Natl Acad Sci USA 99: 10084-10089, 2002.

22. Sun X, Ding H, Hung K and Guo B: A new MALDI-TOF based mini-sequencing assay for genotyping of SNPs. Nucleic Acids Res 28: E68, 2000.

23. Weisenberger DJ, Velicescu M, Cheng JC, Gonzales FA, Liang G and Jones PA: Role of the DNA methyltransferase variant DNMT3b3 in DNA methylation. Mol Cancer Res 2: 62-72, 2004.

24. Liao HK, Su YN, Kao HY, Hung CC, Wang HT and Chen YJ: Parallel minisequencing followed by multiplex matrix-assisted laser desorption/ionization mass spectrometry assay for betathalassemia mutations. J Hum Genet 50: 139-150, 2005.

25. Stanssens P, Zabeau M, Meersseman G, et al: High-throughput MALDI-TOF discovery of genomic sequence polymorphisms. Genome Res 14: 126-133, 2004.

26. Chang KP, Hao SP, Liu CT, et al: Promoter polymorphisms of DNMT3B and the risk of head and neck squamous cell carcinoma in Taiwan: A case-control study. Oral Oncol 43: 345-351, 2007.

27. Bieche I, Tozlu S, Girault I and Lidereau R: Identification of a three-gene expression signature of poor-prognosis breast carcinoma. Mol Cancer 3: 37, 2004.

28. Saito Y, Kanai Y, Sakamoto M, Saito H, Ishii H and Hirohashi S: Overexpression of a splice variant of DNA methyltransferase $3 b$, DNMT3b4, associated with DNA hypomethylation on pericentromeric satellite regions during human hepatocarcinogenesis. Proc Natl Acad Sci USA 99: 10060-10065, 2002.

29. Wang L, Wang J, Sun S, et al: A novel DNMT3B subfamily, $\triangle D N M T 3 B$, is the predominant form of $D N M T 3 B$ in non-small cell lung cancer. Int J Oncol 29: 201-207, 2006.

30. Jinawath A, Miyake S, Yanagisawa Y, Akiyama Y and Yuasa Y: Transcriptional regulation of the human DNA methyltransferase $3 \mathrm{~A}$ and $3 \mathrm{~B}$ genes by Sp3 and Sp1 zinc finger proteins. Biochem J 385: 557-564, 2005.

31. Yanagisawa $Y$, Ito E, Yuasa $Y$ and Maruyama K: The human DNA methyltransferases DNMT3A and DNMT3B have two types of promoters with different $\mathrm{CpG}$ contents. Biochim Biophys Acta 1577: 457-465, 2002. 